\title{
Tratamento da braquimetatarsia por distração osteogênica com fixador externo monolateral
}

\section{Treatment of Brachymetatarsia by Distraction Osteogenesis Using Monolateral External Fixator}

\author{
Adriano Machado Filho ${ }^{1}$ Gabriel Fonseca de Oliveira Costa ${ }^{10}$ Aryell Assis dos Santos Faria ${ }^{10}$ \\ Gustavo Teixeira Leão ${ }^{1}$ Jefferson Soares Martins ${ }^{10}$
}

${ }^{1}$ Departamento de Pé e Tornozelo, Hospital de Urgências de Goiânia Secretaria do Estado da Saúde, Goiânia, GO, Brasil

Rev Bras Ortop 2022;57(1):75-81.
Endereço para correspondência Adriano Machado, B.Sc., Médico Residente em Ortopedia e Traumatologia do Hospital de Urgências de Goiânia - Secretaria do Estado da Saúde, Rua T-37, n. 3000, Ônix Bueno, Setor Bueno, Goiânia, GO, 74230-025, Brasil (e-mail: adrianomf_5@hotmail.com).

\section{Resumo}

\section{Palavras-chave}

- deformidades do pé

- procedimentos ortopédicos

- osteogênese por distração

- ossos do metatarso
Objetivo Descrever o perfil dos pacientes e os resultados obtidos com o uso de fixador externo para alongamento de metatarso em braquimetatarsia.

Métodos Foi realizada uma análise retrospectiva dos prontuários de pacientes com braquimetatarsia tratados entre janeiro de 2018 e abril de 2020. Durante este período, foram operados oito pés de seis pacientes. Foram coletadas e estimadas as frequências em relação a aspectos demográficos e cirúrgicos.

Resultados Todas as pacientes $(100 \% ; n=6)$ eram do sexo feminino, com média de idade de 28 anos, variando de 15 a 48 anos, e motivadas a buscar o serviço de ortopedia em função da deformidade estética. $O$ acometimento era bilateral em duas pacientes e unilateral em quatro pacientes. O tempo médio de alongamento foi de 22 dias ( $\pm 7,15$; intervalo de confiança [IC] 95\%: 19,04-26,81). A velocidade de alongamento foi de $0,5 \mathrm{~mm} /$ dia e o comprimento médio total do alongamento foi de $11,46 \mathrm{~mm}$ ( $\pm 3,57$; IC95\%: 9,52-13,40). Metade das pacientes $(50 \% ; n=3)$ teve infecção local dos pinos e foi tratada com antibióticos; as demais não relataram nenhuma complicação póscirúrgica. As pacientes negaram dor ou calosidade após o procedimento cirúrgico e relataram satisfação com os resultados.

Conclusão Todas as pacientes eram do sexo feminino e buscaram a cirurgia para braquimetatarsia por motivos estéticos. A distração osteogênica a uma taxa de $0,5 \mathrm{~mm} /$ dia resultou em alongamento bem-sucedido do metatarso, com uma baixa frequência de complicações, bons resultados clínicos e alta satisfação das pacientes.

Trabalho desenvolvido no Hospital de Urgências de Goiânia -

Secretaria do Estado da Saúde, Goiânia, GO, Brasil.

recebido

07 de Janeiro de 2021

aceito

02 de Junho de 2021

Publicado on-line

Outubro 1, 2021
DOI https://doi.org/

$10.1055 / \mathrm{s}-0041-1735828$. ISSN 0102-3616. (c) 2021. Sociedade Brasileira de Ortopedia e Traumatologia. All rights reserved.

This is an open access article published by Thieme under the terms of the Creative Commons Attribution-NonDerivative-NonCommercial-License, permitting copying and reproduction so long as the original work is given appropriate credit. Contents may not be used for commercial purposes, or adapted, remixed, transformed or built upon. (https://creativecommons.org/ licenses/by-nc-nd/4.0/)

Thieme Revinter Publicações Ltda., Rua do Matoso 170, Rio de Janeiro, RJ, CEP 20270-135, Brazil 


\begin{abstract}
Keywords

- foot deformities

- orthopedic procedures

- osteogenesis, distraction

- metatarsal bones

Objective To describe the profile of the patients and the results obtained with the use of an external fixator for metatarsal lengthening in brachymetatarsia.

Methods A retrospective analysis of the medical records of patients with brachymetatarsia treated between January 2018 and April 2020 was performed. During this period, eight feet of six patients were operated on. Frequencies were estimated according to demographic and surgical aspects.

Results All patients $(100 \% ; n=6)$ were female, with a mean age of 28 years old, ranging from 15 to 48 years old, and were motivated to seek the orthopedic service due to aesthetic deformity. The deformity was bilateral in two patients and unilateral in four patients. The average lengthening time was 22 days $( \pm 7.15,95 \%$ confidence interval $[\mathrm{Cl}]:$ 19.04-26.81). The lengthening speed was $0.5 \mathrm{~mm} /$ day, and the average total length of the lengthening was $11.46 \mathrm{~mm}( \pm 3.57 ; 95 \% \mathrm{Cl}$ : 9.52-13.40). Half of the patients $(50 \% ; n=3)$ had local infection of the pins and were treated with antibiotics, and the others did not report any postsurgical complications. All patients denied pain or calluses after the surgical procedure and reported satisfaction with the results. Conclusion All patients were female and sought surgery for brachymetatarsia for aesthetic reasons. Osteogenic distraction at a rate of $0.5 \mathrm{~mm} /$ day resulted in successful lengthening of the metatarsal, with a low frequency of complications, good clinical outcomes, and high patient satisfaction.
\end{abstract}

\section{Introdução}

A braquimetatarsia é uma deformidade congênita rara, predominante no sexo feminino $(92,5 \%),{ }^{1}$ na qual ocorre o encurtamento do metatarso do pé em $\geq 5 \mathrm{~mm}$ em relação ao arco transversal do pé. ${ }^{2}$ A braquimetatarsia afeta principalmente o quarto metatarso, mas a patologia pode ocorrer em qualquer osso metatarsal. Além do incomodo estético, a deformidade pode prejudicar o mecanismo de sustentação do pé, causando dor à deambulação. O tratamento cirúrgico é uma alternativa na busca de um resultado estético e funcional satisfatório. ${ }^{3,4}$

A etiologia da patologia ainda não está completamente esclarecida. A principal causa é o fechamento prematuro da placa de crescimento metatarsal, decorrente de fatores hereditários, pós-traumáticos ou pós-cirúrgicos. ${ }^{5} \mathrm{~A}$ braquimetatarsia pode estar associada a doenças como síndrome de Down, síndrome de Apert, osteodistrofia de Albright, anemia falciforme, anomalia diastrófica, poliomielite, endocrinopatias e síndrome de Turner. ${ }^{1}$ Outras causas secundárias descritas na literatura incluem infecções, tumores e exposição à radiação. $^{6}$

A braquimetatarsia é tipicamente detectada entre 1 e 5 anos de idade, pode afetar um ou mais metatarsos e ser unilateral ou bilateral. A incidência de bilateralismo acontece em $>50 \%$ dos casos descritos na literatura. ${ }^{7}$ As queixas dos pacientes incluem metatarsalgia, calos, irritação no uso de calçados e contraturas de tecidos moles. No entanto, a aparência estética é a principal queixa em mulheres jovens, decorrente do encurtamento do raio afetado ou de deformidades associadas dos outros dedos dos pés, incluindo o desvio dos raios normais para preencher a lacuna formada pelo metatarso comprometido, gerando deformidades em valgo nos dedos localizados medialmente e em varo nos dedos localizados lateralmente. ${ }^{8}$

O tratamento cirúrgico da braquimetatarsia inclui técnicas que utilizam alongamento agudo com uso de enxerto ósseo interposicional ou alongamento gradual por distração osteogênica (com ou sem enxerto), associados ou não a procedimento em falanges adjacentes. ${ }^{5,9,10}$ A osteogênese por distração usando fixadores externos é uma técnica amplamente utilizada, principalmente quando alongamentos $>1 \mathrm{~cm}$ são necessários. ${ }^{11}$ Embora a longa duração do tratamento e o entendimento e cooperação do paciente possam parecer desfavoráveis na escolha desta técnica, ${ }^{8}$ algumas vantagens importantes do uso da distração gradual incluem o aumento da estabilidade da construção, possibilidades de alongamentos maiores, maior controle do alongamento, menor tempo cirúrgico e ausência de necessidade de enxerto ósseo (o que pode gerar dor e desconforto na área doadora, além do risco de absorção do enxerto). ${ }^{1}$

O objetivo do presente estudo foi descrever o perfil dos pacientes e os resultados obtidos com o uso de fixador externo para alongamento de metatarso em braquimetatarsia em um serviço de ortopedia e traumatologia.

\section{Material e Métodos}

O presente estudo de caráter retrospectivo com análise dos prontuários foi aprovado pelo Comitê de Ética em Pesquisa da instituição proponente com o número CAEE 34810920.2.0000.0033. Os dados coletados foram avaliados nos programas Microsoft Excel versão 2007 (Microsoft Corporation, Redmond, WA, EUA) e no programa estatístico SPSS 

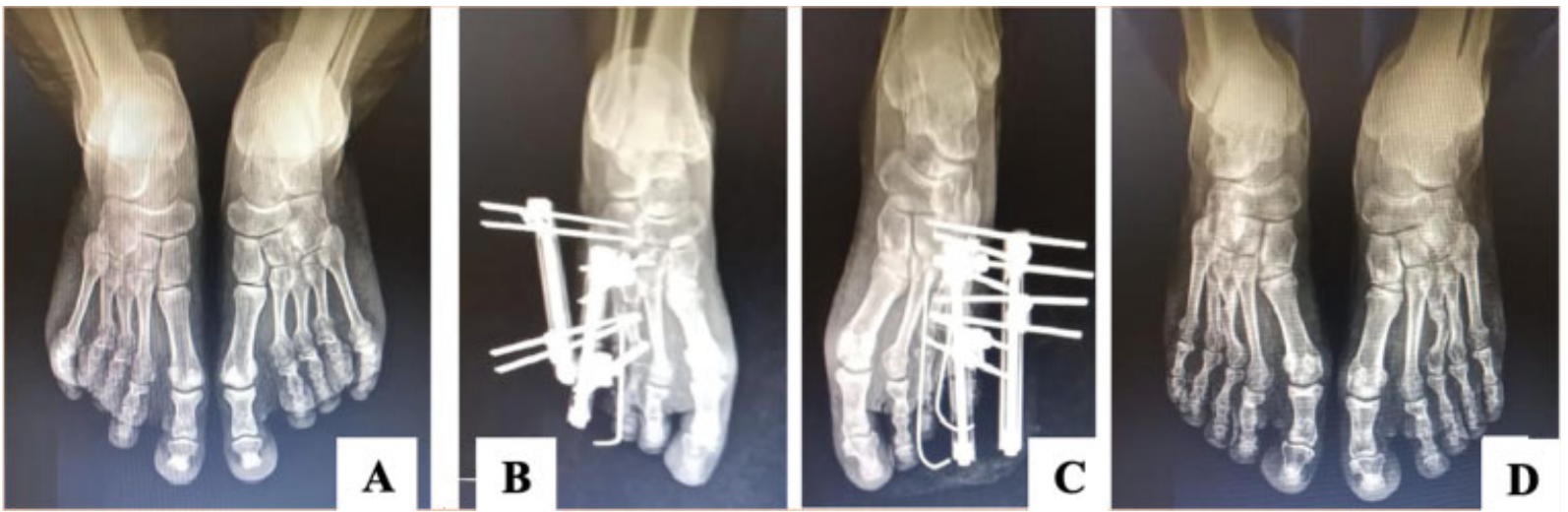

Fig. 1 Radiografia em anteroposterior dos pés com carga demonstrando evolução de paciente com braquimetatarsia bilateral em $3^{\circ} \mathrm{e} 4^{\circ}$ metatarsos submetido a distração osteogênica. (A) radiografia pré-operatória, (B) radiografia pós-operatória do lado direito, (C) radiografia pósoperatória do lado esquerdo e (D) resultado após 12 semanas de tratamento.

Statistics for Windows versão 17.0 (SPSS Inc., Chicago, IL, EUA). 0 estudo incluiu 6 pacientes com diagnóstico de braquimetatarsia tratadas entre janeiro de 2018 e abril de 2020 e um total de 8 pés e 13 metatarsos.

Foram avaliadas as seguintes variáveis epidemiológicas: idade, gênero, motivo da consulta (deformidade estética, metatarsalgia, dificuldade em calçar sapatos), lado acometido, metatarso acometido, presença de dor ou calosidade (pré- e pós-cirurgia), tempo de distração do fixador, alongamento obtido (em mm), tempo de uso de fixador, complicações e satisfação pós-cirúrgica.

Utilizou-se radiografias (-Figura $\mathbf{1}$ ) nas incidências em anteroposterior e perfil para avaliação dos parâmetros de alongamento (ganho de comprimento) e de calogênese do regenerado ósseo. 0 aspecto clínico desejado na correção da deformidade determinou o período do alongamento a ser realizado pela paciente via ambulatorial.

Os critérios de inclusão foram pacientes submetidas a distração osteogênica com fixador externo para alongamento de metatarso em braquimetatarsia. Foram excluídas da amostra as participantes que foram submetidas a qualquer outro procedimento cirúrgico para alongamento metatarsal que não a distração osteogênica com fixador externo monolateral.

A técnica cirúrgica utilizada seguiu a seguinte padronização: paciente em decúbito dorsal com inclinação lateral de $45^{\circ}$ no lado a ser operado, com uso de garrote pneumático inflado a $300 \mathrm{mmHg}$. Sob orientação de fluoroscopia, dois pinos de Schanz são inseridos na metáfise proximal e dois na metáfise distal. Os pinos são posicionados em uma inclinação de $45^{\circ}$ em relação ao eixo do metatarso a ser alongado, após incisão de pele com bisturi, lâmina número 15 , dissecção de partes moles com pinça hemostática delicada para afastamento dos tendões e broqueamento com perfurador com controle rotacional. Quando o metatarsiano a ser alongado apresenta-se muito encurtado, aceita-se a colocação de um pino no osso cuneiforme correspondente. Após a instalação e o travamento dos dois cabeçais do minifixador, é feita uma incisão de pele com dissecção por planos, exposição da diáfise e múltiplas perfurações com uma broca delicada para auxiliar na corticotomia. É realizada corticotomia com osteótomos delicados sob visualização direta e é feita a conferência da mesma por fluoroscopia no intraoperatório. Em seguida, realiza-se a fixação da articulação metatarsofalângica com um fio de Kirschner 1.5 (- Figura 2), para se evitar deformidade em flexão da falange durante o alongamento por distração. Depois, faz-se o fechamento da incisão de pele no local da corticotomia com fio de nylon 4.0 e curativos em pinos. Quando há a necessidade de colocação de mais de um fixador externo, o procedimento é realizado da mesma maneira, tomando-se o cuidado na distância entre as montagens para não dificultar a distração e o alongamento.

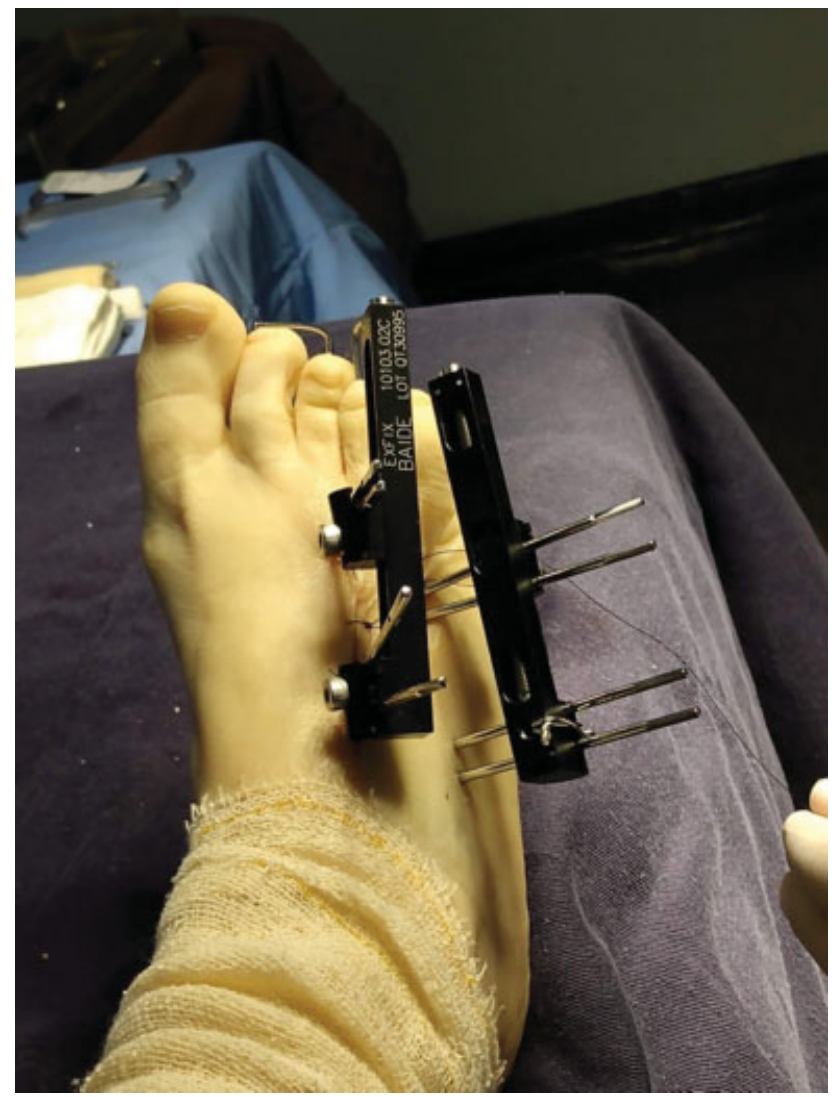

Fig. 2 Fixador externo monolateral instalado para distração óssea em inclinação de $45^{\circ}$ e fios de Kirschnner intramedulares. 
Tabela 1 Dados demográficos dos pacientes (pré-operatório)

\begin{tabular}{|l|l|}
\hline Dados & Valores \\
\hline Número de participantes $(n)$ & 6 \\
\hline Número de metatarsos $(n)$ & 13 \\
\hline Número de pés $(n)$ & 8 \\
\hline Sexo feminino $(n)$ & 6 \\
\hline Idade (média \pm desvio padrão) & $28 \pm 14,62$ anos \\
\hline Lateralidade $(n)$ & - \\
\hline Bilateral & 2 pacientes \\
\hline Direito & 2 pacientes \\
\hline Esquerdo & 2 pacientes \\
\hline Pacientes com insatisfação estética & $6(100 \%)$ \\
\hline
\end{tabular}

As pacientes do estudo receberam alta hospitalar em 48 horas, sendo orientadas sobre quando iniciar e como realizar a distração óssea, $0,25 \mathrm{~mm}$ a cada 12 horas, com realização de radiografias semanais e carga parcial com sola rígida. A retirada do fixador externo foi realizada em todas as pacientes na $12^{a}$ semana pós-operatória, sob sedação.

\section{Resultados}

No período de 2018 a 2020, foram tratadas cirurgicamente seis pacientes, com um total de 8 pés e 12 metatarsos ( - Tabela 1) submetidos a distração osteogênica com fixador externo. A média de idade foi de 28 anos ( $\pm 14,62$; intervalo de confiança [IC] 95\%: 16,31-39,69), variando de 15 a 48 anos. Todas as pacientes $(100 \% ; n=6)$ eram do sexo feminino, com braquimetatarsia congênita, e foram motivadas a buscar o serviço de ortopedia em função da deformidade estética. Apenas uma paciente queixava-se de dor pré-cirúrgica.
0 acometimento era bilateral em duas pacientes e unilateral em quatro pacientes. O tempo médio de alongamento foi de 22 dias ( $\pm 7,15$; IC95\%: 19,04-26,81), e o comprimento médio total do alongamento foi de $11,46 \mathrm{~mm}$ ( $\pm 3,57$; IC95\%: 9,52-13,40); a média foi maior no pé direito $(25,43 \pm 9,07$ dias $)$ em comparação com o esquerdo $(20,00 \pm 2,19$ dias; $p<0,05)$. Independente do lado acometido, a frequência de deformidade é maior no quarto metatarso, no qual todas as pacientes tinham deformidade (-Figura 3).

Na - Tabela 2, estão apresentados os dados individuais referentes ao tempo de alongamento (em dias), o comprimento obtido (em $\mathrm{mm}$ ) e o tempo de acompanhamento (em meses) das pacientes tratadas com uso de fixador externo separados em função do lado e do metatarso acometidos. Os metatarsos do lado direito apresentaram as maiores médias de tempo de alongamento e comprimento obtido. Para o quarto metatarso do lado direito, o alongamento durou em média 28,76 dias e o comprimento médio obtido foi de $14,33 \mathrm{~mm}$. Já para o quarto metatarso do lado esquerdo, a média foi de 22 dias e o comprimento foi de $11 \mathrm{~mm}$. 0 terceiro metatarso seguiu a mesma tendência, com um tempo de alongamento médio do lado direito de 24,67 dias e um comprimento médio $12,33 \mathrm{~mm}$, superiores aos do lado esquerdo, de 18 dias e $9 \mathrm{~mm}$. A velocidade de alongamento foi de $0,5 \mathrm{~mm} / \mathrm{dia}$, e as pacientes foram orientadas a alongar diariamente o fixador. 0 tempo médio de acompanhamento foi de 18 meses, variando de 6 a 48 meses.

De acordo com os dados dos prontuários, $50 \%(n=3)$ das pacientes tiveram infecção local dos pinos e foram tratadas com antibióticos; as demais não relataram nenhuma complicação pós-cirúrgica. Todas as pacientes negaram dor ou calosidade após o procedimento cirúrgico e relataram estarem satisfeitas com os resultados obtidos.

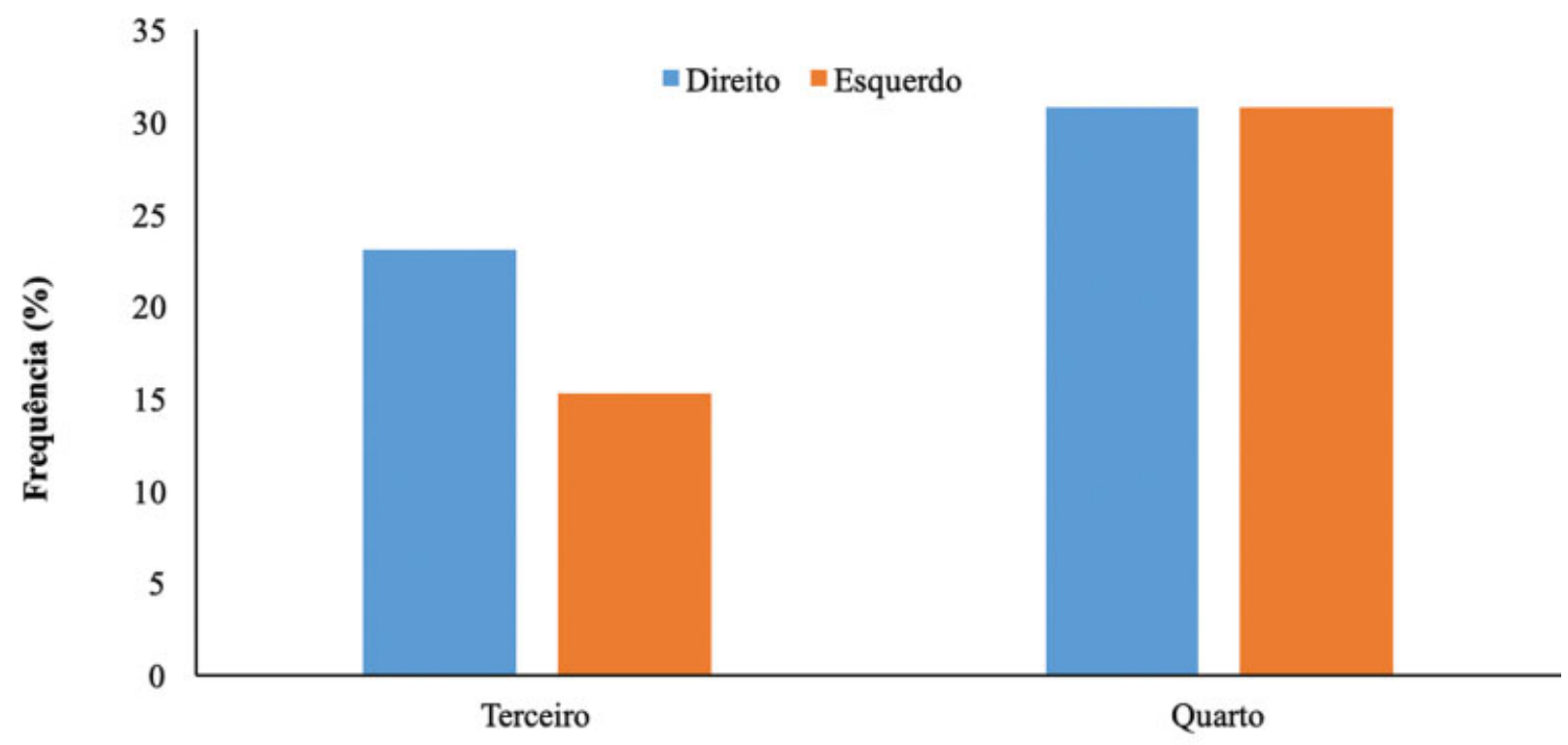

Metatarso

Fig. 3 Frequência da braquimetatarsia de acordo com o lado e o metatarso acometido em pacientes em uso de fixador externo monolateral para alongamento. 
Tabela 2 Dados das pacientes com braquimetatarsia quanto ao tempo de alongamento ( $\mathrm{T}$ ), comprimento obtidos (C) e tempo de acompanhamento (TA) submetidas ao tratamento com uso de fixador externo

\begin{tabular}{|c|c|c|c|c|c|c|c|c|c|}
\hline \multirow{3}{*}{\begin{tabular}{|l|} 
Lado \\
Metatarso* \\
Paciente
\end{tabular}} & \multicolumn{4}{|l|}{ Direito } & \multicolumn{4}{|c|}{ Esquerdo } & \multirow{3}{*}{$\begin{array}{l}\text { TA } \\
\text { (meses) }\end{array}$} \\
\hline & \multicolumn{2}{|l|}{ Terceiro } & \multicolumn{2}{|l|}{ Quarto } & \multicolumn{2}{|l|}{ Terceiro } & \multicolumn{2}{|l|}{ Quarto } & \\
\hline & $\mathrm{T}$ (dias) & $\mathrm{C}(\mathrm{mm})$ & $\mathrm{T}$ (dias) & $\mathrm{C}(\mathrm{mm})$ & $\mathrm{T}$ (dias) & $C(\mathrm{~mm})$ & $\mathrm{T}$ (dias) & $\mathrm{C}(\mathrm{mm})$ & \\
\hline 1 & - & - & - & - & - & - & 20 & 10 & 24 \\
\hline 2 & - & - & 18 & 9 & - & - & - & - & 48 \\
\hline 3 & - & - & - & - & - & - & 20 & 10 & 12 \\
\hline 4 & 18 & 9 & 20 & 10 & 18 & 9 & 20 & 10 & 8 \\
\hline 5 & 20 & 10 & 26 & 13 & 18 & 9 & 24 & 12 & 12 \\
\hline 6 & 36 & 18 & 40 & 20 & - & - & - & - & 6 \\
\hline Média \pm DP & $\begin{array}{l}24,67 \pm \\
9,87\end{array}$ & $\begin{array}{l}12,33 \pm \\
4,93\end{array}$ & $\begin{array}{l}28,67 \pm \\
9,93\end{array}$ & $\begin{array}{l}14,33 \pm \\
4,97\end{array}$ & $\begin{array}{l}18,00 \pm \\
0,00\end{array}$ & $\begin{array}{l}9,00 \pm \\
0,00\end{array}$ & $22,00 \pm 2,00$ & $11,00 \pm 1,00$ & $\begin{array}{l}18,3 \pm \\
15,8\end{array}$ \\
\hline
\end{tabular}

Abreviações: C, comprimento; DP, desvio padrão; T, tempo; TA, tempo de acompanhamento.

\section{Discussão}

A principal queixa dos pacientes que buscam tratamento para a braquimetatarsia está associada a deformidade estética ou dificuldade funcional. ${ }^{2,6}$ Estes pacientes geralmente se excluem das atividades sociais e esportivas devido à aparência do pé. ${ }^{12}$ No presente estudo, a aparência estética foi a principal queixa relatada pelas pacientes submetidas ao tratamento cirúrgico. Cerca de $25 \%$ do estresse primário ao suporte de peso é absorvido pelos metatarseanos. 0 encurtamento de qualquer um deles leva à sobrecarga e à frouxidão do ligamento transverso, ocasionando um contato inadequado do antepé, pressão excessiva e comprometimento da função da musculatura, resultando em dor e fadiga na perna e no pé. ${ }^{13} \mathrm{~A}$ dor é considerada a segunda indicação mais comum para o tratamento cirúrgico da braquimetatarsia. ${ }^{14}$ Nenhuma das pacientes do presente estudo relatou queixas álgicas importantes.

A maioria dos casos descritos na literatura ${ }^{3,4,8,9}$ e todos os avaliados no presente trabalho são do sexo feminino. A braquimetatarsia é uma deformidade congênita rara (a incidência na população varia entre 0,02 e 0,05\%), com maior frequência no sexo feminino na razão de 25:1 em relação ao sexo masculino. ${ }^{12}$

A maior procura pelo tratamento cirúrgico da braquimetatarsia ocorre na adolescência, em virtude da insatisfação estética. $^{2}$ Porém, no presente estudo, metade da amostra obtida era formada por adolescentes e a outra metade por adultos jovens ( $50 \% ; n=3$ ). A média de idade das pacientes operadas foi de 28 anos, próxima à observada por Giannini et al. $^{8}$ (média de 27 anos, variando de 12 a 42 anos) ao avaliarem 29 pacientes operados para correção de braquimetatarsia em um hospital italiano por 10 anos. Estes mesmos autores ${ }^{8}$ sugerem que a cirurgia seja feita após os 12 anos de idade, pois deve-se aguardar a conclusão do crescimento ósseo, além da dificuldade de manejo após a cirurgia em crianças menores. Lee et al. ${ }^{15}$ relatam um menor índice de complicações em adolescentes operados quando comparados com adultos em relação à consolidação do regenerado ósseo.

Lamm ${ }^{11}$ sugere que, para um ganho de $20 \mathrm{~mm}$ no alongamento do metatarso, o tempo necessário seria de $\sim 45$ dias, sendo 5 dias de latência e 40 dias de distração a uma velocidade de $0,5 \mathrm{~mm} / \mathrm{dia}$. No presente estudo, a velocidade de distração foi de $0,5 \mathrm{~mm} / \mathrm{dia}(0,25 \mathrm{~mm}$ de 12 em 12 horas $) \mathrm{e}$ o tempo médio de alongamento foi de 22 dias. Velocidades de alongamento $>1 \mathrm{~mm} /$ dia podem resultar em dor, tensão excessiva nos tecidos moles e luxação articular. ${ }^{16} \mathrm{O}$ alongamento gradual em um único estágio reduz o risco de comprometimento neurovascular, uma vez que gera menor tensão dos tecidos moles. Quando a necessidade de alongamento é $>10 \mathrm{~mm}$, o risco de comprometimento neurovascular é a principal limitação do tratamento agudo com alongamento, enxerto ósseo e fixação interna. ${ }^{11}$

Ao avaliarem 48 casos de braquimetatarsia, Peña-Martínez et al. ${ }^{16}$ observaram que o quarto metatarso era o mais acometido, representando $98 \%$ dos casos, e o terceiro metatarso os outros $2 \%$. No presente estudo, $61,5 \%(n=8)$ das deformidades eram no quarto metatarso e $38,5 \%(n=5)$ no terceiro metatarso ( - Figura 3). A técnica utilizada empregou o uso de fixador externo monolateral associado ao uso de fios de Kirschner (-Figuras 2 e 4 ), promovendo estabilidade na articulação metatarso-falangeana e evitando deformidade em flexão do metatarso durante o alongamento ósseo.

A distração osteogênica é o tratamento mais aceito e mais bem-sucedido realizado por cirurgiões de pé e tornozelo para alongamento do metatarso. ${ }^{17}$ As vantagens do alongamento gradual são a capacidade de obter um comprimento maior em relação ao obtido pelo uso de enxerto ósseo intercalar, permitir o suporte de peso imediato, preservando o movimento da articulação metatarso-falângica, e o fato de não apresentar necessidade de enxerto ósseo, o que pode gerar desconforto em área doadora. ${ }^{11}$ Tais vantagens tornam esta técnica de distração gradual o método mais utilizado quando se busca um alongamento $>15 \mathrm{~mm}$ ou $>25 \%$ do tamanho inicial do metatarso. ${ }^{1}$ 

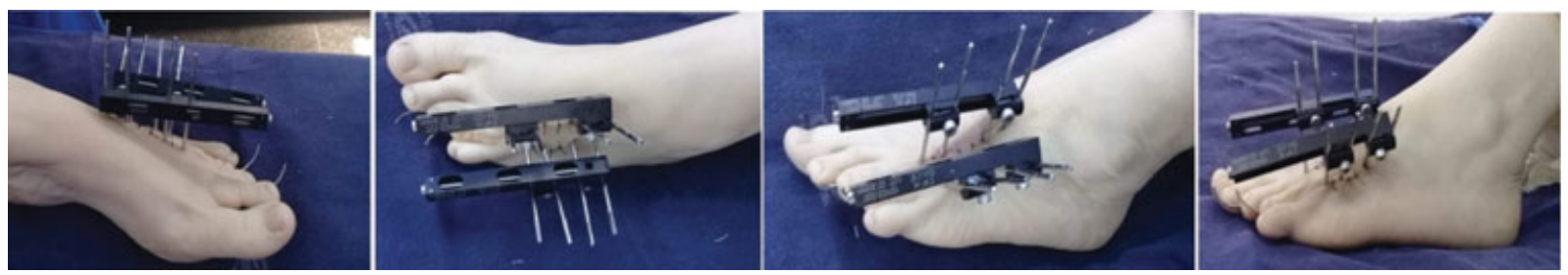

Fig. 4 Pós-operatório de paciente com deformidade em $3^{\circ}$ e $4^{\circ}$ metatarsos do pé esquerdo submetidos a alongamento ósseo com fixador externo monolateral.
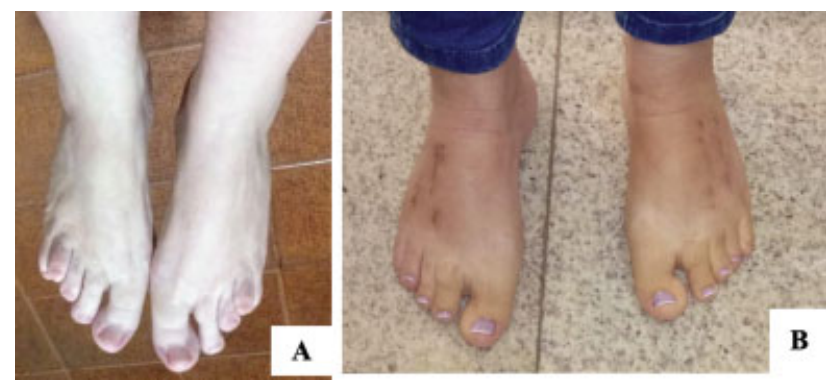

Fig. 5 Aspecto clínico pré-operatório (A) e pós-operatório (B) de paciente com braquimetatarsia bilateral em $3^{\circ}$ e $4^{\circ}$ metatarsos submetidos a distração osteogênica com uso de fixador monolateral.

A distração osteogênica tem como desvantagem a necessidade do ajuste regular do fixador externo para alongamento, a possibilidade de formação óssea insuficiente e o risco de infecção local. Para estímulo da formação óssea adequada, uma osteotomia cuidadosa deve ser realizada com o mínimo de dano ao tecido mole e vascular. ${ }^{11,18}$

No alongamento por distração osteogênica em 2 estágios, a distração é realizada mais rapidamente ( $\sim 2 \mathrm{~mm}$ ao dia) e, após alcançado o comprimento desejado, é feita a colocação de enxerto ósseo no regenerado e a substituição da fixação externa pela interna, na ausência de infecção dos pinos, com o objetivo de diminuir o tempo do fixador externo e a rigidez articular. $^{14}$

Masada et al. ${ }^{19}$ relatam maior número de complicações pós-operatórias quando o alongamento realizado é $>40 \%$ do comprimento original do metatarso, citando alongamento excessivo, rigidez articular, perda de alinhamento, retardo de consolidação e fratura do regenerado.

Em todas as pacientes analisadas, o alongamento alcançado foi obtido sem soltura dos pinos, com um bom regenerado ósseo, sem a necessidade do uso de enxerto em um segundo tempo ou de outro procedimento cirúrgico além da retirada do fixador externo e do fio de Kirschner via ambulatorial. A consolidação fora obtida em $100 \%$ das pacientes dentro do período 8 a 12 semanas. Ao fim do tratamento, todas as pacientes apresentaram boa correção cirúrgica, bom aspecto estético (-Figura 5), alto grau de satisfação com a cirurgia, e nenhum grau de rigidez ou déficit de mobilidade nas articulações dos terceiro e quarto dedos dos pés.

Na literatura científica, está bem estabelecido que a fixação externa restaura o comprimento do metatarso, a posição dos dedos dos pés, a função do pé e dos dedos, melhora a cosmese e reduz a dor. ${ }^{20}$ Os resultados obtidos no presente estudo foram comparáveis aos de outras pesquisas em termos de características demográficas, quantidade de correção cirúrgica e resultados clínicos. ${ }^{9,20,21}$ O presente estudo também apresenta algumas limitações em função da sua natureza retrospectiva, além de possuir uma amostra relativamente pequena e um curto tempo de seguimento no pós-operatório.

\section{Conclusão}

Todas as pacientes eram do sexo feminino e buscaram a cirurgia para braquimetatarsia por motivos estéticos. A distração osteogênica gradual a uma taxa de $0,5 \mathrm{~mm} / \mathrm{dia}$ com uso de fixador externo e fixação da articulação metatarso-falângica com fio de Kirschner resultou em alongamento bem-sucedido do metatarso, com bons resultados estéticos e funcionais. Apesar das limitações da presente pesquisa, os bons resultados clínicos e a alta satisfação das pacientes tornam o uso de fixador externo monolateral para alongamento de metatarso em braquimetatarsia uma boa opção.

\section{Suporte Financeiro}

Não houve suporte financeiro de fontes públicas, comerciais, ou sem fins lucrativos.

Conflito de Interesses

Os autores declaram não haver conflito de interesses.

\section{Referências}

1 Rocha FA, Mansur H, Meira RB, Gusmão L, Gonçalves CB, Castro Júnior IM. One-stage correction of multiple brachymetatarsia and hallux valgus with calcaneal autograft. Sci J Foot Ankle 2018;12 (04):342-346

2 Kim HT, Lee SH, Yoo CI, Kang JH, Suh JT. The management of brachymetatarsia. J Bone Joint Surg Br 2003;85(05):683-690

3 Smolle E, Scheipl S, Leithner A, Radl R. Management of congenital fourth brachymetatarsia by additive autologous lengthening osteotomy (AALO): a case series. Foot Ankle Int 2015;36(03): 325-329

4 Desai A, Lidder S, Armitage AR, Rajaratnam SS, Skyrme ADS. Brachymetatarsia of the fourth metatarsal, lenghtening scarf osteotomy with bone graft. Ortop Rev (Pavia) 2013;5(03):e21

5 Lara LCR, Franco Filho N, Marques AFS. Tratamento cirúrgico da braquimetatarsia. Rev ABTPe 2008;2(01):7-11

6 Lee WC, Suh JS, Moon JS, Kim JY. Treatment of brachymetatarsia of the first and fourth ray in adults. Foot Ankle Int 2009;30(10): 981-985

7 Scher DM, Blyakher A, Krantzow M. A modified surgical technique for lengthening of a metatarsal using an external fixator. HSS J 2010;6(02):235-239 
8 Giannini S, Faldini C, Pagkrati S, Miscione MT, Luciani D. Onestage metatarsal lengthening by allograft interposition: a novel approach for congenital brachymetatarsia. Clin Orthop Relat Res 2010;468(07):1933-1942

9 Lee KB, Yang HK, Chung JY, Moon ES, Jung ST. How to avoid complications of distraction osteogenesis for first brachymetatarsia. Acta Orthop 2009;80(02):220-225

10 Iida N, Watanabe A. A new surgical procedure for brachymetatarsia by a hydroxyapatite graft. Eur J Plast Surg 2013;36:41-44

11 Lamm BM. Percutaneous distraction osteogenesis for treatment of brachymetatarsia. J Foot Ankle Surg 2010;49(02):197-204

12 Shim JS, Park SJ. Treatment of brachymetatarsia by distraction osteogenesis. J Pediatr Orthop 2006;26(02):250-254

13 Barik S, Farr S. Brachymetacarpia and brachymetatarsia: do we need to operate? EFORT Open Rev 2021;6(01):15-23

14 Shecaira AP, Fernandes RMP. Brachymetatarsia: One-stage Versus Two-Stage Procedures. Foot Ankle Clin 2019;24(04):677-687

15 Lee KB, Park HW, Chung JY, Moon ES, Jung ST, Seon JK. Comparison of the outcomes of distraction osteogenesis for first and fourth brachymetatarsia. J Bone Joint Surg Am 2010;92(16):2709-2718
16 Peña-Martínez VM, Palacios-Barajas D, Blanco-Rivera JC, et al. Results of External Fixation and Metatarsophalangeal Joint Fixation With K-Wire in Brachymetatarsia. Foot Ankle Int 2018;39 (08):942-948

17 Lamm BM, Gourdine-Shaw MC. Problems, obstacles, and complications of metatarsal lengthening for the treatment of brachymetatarsia. Clin Podiatr Med Surg 2010;27(04):561-582

18 Hurst JM, Nunley JA II. Distraction osteogenesis for the shortened metatarsal after hallux valgus surgery. Foot Ankle Int 2007;28 (02):194-198

19 Masada K, Fujita S, Fuji T, Ohno H. Complications following metatarsal lengthening by callus distraction for brachymetatarsia. J Pediatr Orthop 1999;19(03):394-397

20 Lee WC, Yoo JH, Moon JS. Lengthening of fourth brachymetatarsia by three different surgical techniques. J Bone Joint Surg Br 2009; 91(11):1472-1477

21 Fusini F, Langella F, Catani O, Sergio F, Zanchini F. Mini-invasive treatment for brachymetatarsia of the fourth ray in females: percutaneous osteotomy with mini-burr and external fixation-a case series. J Foot Ankle Surg 2017;56(02):390-394 